Article

\title{
Fish and Food Security: Potential for Global Fishery Collapse
} Peter Jacques

Department of Political Science, University of Central Florida, 4000 Central Florida Blvd, Orlando, Florida, 32816/ Peter.Jacques@ucf.edu

Tel.: +1-407-823-2608; Fax: +1-407-823-0051

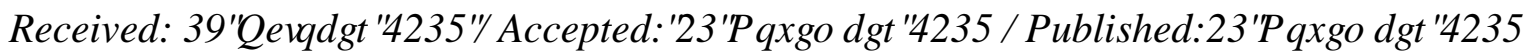

\begin{abstract}
Around the world fisheries are being stressed through a host of disturbances, not least of which is fishing, which substantially increased in the post-war period compared to prior human experience through history. Single-species stocks have collapsed in the past, but is it possible, even if improbable, that there could be an systematic collapse of global fisheries? The first part of this paper asks whether or not global fisheries could be described accurately as a panarchy of subsidiary adaptive systems in which global fisheries the highest level and scale, or if fisheries are disparate populations that cannot accurately be described as making up a coherent system through a series of teleconnections and interdependencies. If global fisheries can be described as the highest scale in fishery systems, then we should be able to analyze the potential of global collapse according to the adaptive cycle. If we cannot show the mechanisms and relationships that would indicate a global system, what would be highest level in fishery systems and what are the conditions of these varied levels? To advance thinking in these terms, the paper analyzes relevant variables, such as habitat changes in the World Ocean System, important to global fisheries to get an indication of directions, as well as historical collapses to understand and potentially build a social-ecological model of global fishery collapse.
\end{abstract}

Keywords: Fish, Fishery Management, Fishery Collapse, Food Security

\section{Introduction}

This paper questions whether or not it makes sense to conceptualize global fisheries as a global complex system-specifically, are global fisheries a nested, hierarchical complex system, or a panarchy (Gunderson and Holling, 2002) and if so, what are the directions of important variables in this hypothetical system? Complex systems are sometimes related to the idea of chaos, where there are so many variables that there appears to be no order except post hoc, and if fisheries are complex systems, global or not, then it will take planning and prudence if the inevitable changes are to be more surprises and less crises (Berkes et al., 1998, Folke, 2006, Folke et al., 2004, Scheffer et al., 2001, Walker et al., 2006). Part one of this set address the biological oceanography relevant to a global fish 
panarchy, and part two addresses the "social oceanography" of such a system that include issues like the political economic history, the changes to fishing fleet structures, policy, and similar pressures and adaptation that must be considered in any fishery research because a fishery is itself a network of social effort to catch part of a fish population.

The answer to this question is important because if fisheries are plausibly a globally interconnected complex system, it is theoretically plausible, if not likely at some unpredictable time, for a global collapse event that could unfold quickly, such as over a decadal time period. It would also be plausible that integrated goals for global fishery resilience would make sense, and in this case it may make sense to think of a globally coordinated fishery regime (Barkin and DeSombre, 2013a, Barkin and DeSombre, 2013b). The stakes are obviously high because fish provide a very important source of food, but the wild fish catch is not expected to keep up with demand, specifically threatening food security for the world's poorest peoples (Godfray et al., 2010, Kent, 1997, Pauly et al., 2005, Coulthard et al., 2011). One analysis in ICES Journal of Marine Science, explains that the production of fish must increase by 50\% to meet expected demands for food (Pauly and Palomares, 2005), though affluent countries have been able to and will continue to be able to (to lesser and lesser degrees) substitute lost local fisheries for imports(Pauly et al., 2005, Jacques, 2006). Of course, fish are also a vital source of revenue and jobs in direct landings that value between $\$ 80-\$ 85$ billion annually (Willman et al., 2008); and, economic impact beyond just landings including indirect and induced economic impact, world fisheries produce \$225 to \$240 billion annually (Dyck and Sumaila, 2010)-even though over half of the landed value is lost due to mismanagement(Arnason, 2011). Finally, fish and fishing play a crucial role in human meaning and culture and have done so since the very old coastal cultures (Jacques, 2009).

The notions here are explicitly inter-disciplinary and are carried out under the auspices of "social oceanography" - or the study of integrated social-marine systems (Jacques, 2010b). This interdisciplinarity is essential for the question at hand because the direction of the world's fisheries are deeply tied to the biophysical conditions of the ocean and fish, and the behavior and context of human activity - and not just fishing. In the end, if there is a global fishery system, we must have a nuanced view of human behavior in this system.

\section{Panarchy Theory and Fisheries}

Panarchies are hierarchical adaptive cycles. The adaptive cycle is a theory proposed first by C.S. 'Buzz' Hollings (1973) regarding the resilience and vulnerability of ecosystems. The theory has since been extensively expanded and developed mainly by the Resilience Alliance and is known to apply to varied social-ecological systems (Holling, 2001, Gunderson and Holling, 2002, Folke et al., 2004). Adaptive cycles have four phases: growth, conservation, release/collapse, and reorganization. The idea of interest for fisheries is that after the growth in fishing, fish populations hit a point of conservation where growth slows and less fish are caught with the same effort. If pressure continues, the population will cross a threshold where the population collapses.

"When a level in the panarchy enters its $\Omega$ phase of creative destruction, the collapse can cascade to the next larger and slower level by triggering a crisis. Such an event is most likely if the slower level is 
at its $\mathrm{K}$ phase, because at this point the resilience is low and the level is particularly vulnerable" (Hollings 2001, 398). More simple and clear terminology is offered by Marten (2013a) that refers to these same cycles as "growth," "equilibrium," "dissolution (or collapse)," and "reorganization."

Further, at the global level beyond LMEs, Garcia and Charles (2007) write that, "Supplementing a detailed analysis by Garcia (1992), the long-term evolution of world fisheries might be described using the cross-loop 'figure of eight' model," citing Gunderson and Holling's (2002) edited volume, Panarchy (emphasis added). Specifically, Garcia and Charles describe the stages of the (r) phase as fisheries moved from artisanal to industrial fishing (1900-1945) (WWII provided a well-known respite for Atlantic fisheries), with development growing 1945-1960, and steep expansion from 1960-1985. They indicate that the "global fisheries crisis" had been building up the entire century, indicating that world fisheries entered into the $\mathrm{K}$ phase.

Thus, from the literature, we can say that fisheries theoretically exist within a nested hierarchy of complex adaptive systems from local, LME, and global scales. The next question is - what are the important variables that affect fisheries at the global level to either make them more vulnerable or resilient?

\section{The Future of Fisheries}

Figure 1 shows the well-known global fish catch pattern, and we must ask - where is this curve going? There are only three options: it can plateau, increase, or decrease. If fisheries are complex systems, changes in populations will likely be non-linear shifts between stable states but the shape of the curve can take several forms [1]. Holt[2], in a critique of a World Bank [3] report, comments on the expected growth curves of the "total biomass of all exploited species" (emphasis in original, p. 4). Here he notes that many fisheries studies have arbitrarily used a simple logarithmic curve, which:

...is achieved by assuming that the maximum sustainable yield (MSY), by weight, of all commercial exploited fish stocks, lumped together, is somewhat more than the average recent annual reported catch (taken to be 85.7 million tonnes in the 'base year'... by an amount taking some account of estimates of Illegal, Unregulated and Unreported (IUU) catches and of fish discarded at sea for one reason or another; the MSY is assumed to be 95 million tonnes. Given the logistic curve this would be obtained when the total biomass was one half of the carrying capacity (the pre-exploited equilibrium level), which is taken to be 453 million tonnes (Holt, p. 4).

Holt calls these actual numbers into question, arguing that the pristine populations would be much larger than assumed, and that MSY thresholds are themselves arbitrary (see Part II of this series of articles for more detail). He doubts that logistic or Fox, also called Pella-Tomlinson curves, accurately represent MSY, and if MSY can be inferred from catch data against projected models of pristine biomass as is done in the World Bank report and elsewhere.

Figure 1 marks global reported catch with three trend lines: exponential, logarithmic, and polynomial. The exponential most closely relates to Fox-based MSY, the logarithmic relates Schaeffer MSY 
curves, and polynomial trends are those with more fluctuations. The Fox model (red) explains the variance in the graph the least. The simple logarithmic curve (black) explains $89 \%$ of the variance, but the polynomial line (blue) explaining the curve almost perfectly $\left(r^{2}=.98\right)$. Consequently, the peak of this curve is flatter than logistic Schaeffer expectations, but not by much and if we imagine Figure 1 as one half of the picture, the other half very likely will see a decline, and if it is a panarchy, the decline is likely to be catastrophic - that is, rapid and consequential to a new state. Thus, if the past is like the future, fisheries may experience collapse in coming decades.

Figure 1. Global Marine Capture Fisheries Curve from Fishstat 2.0[4]

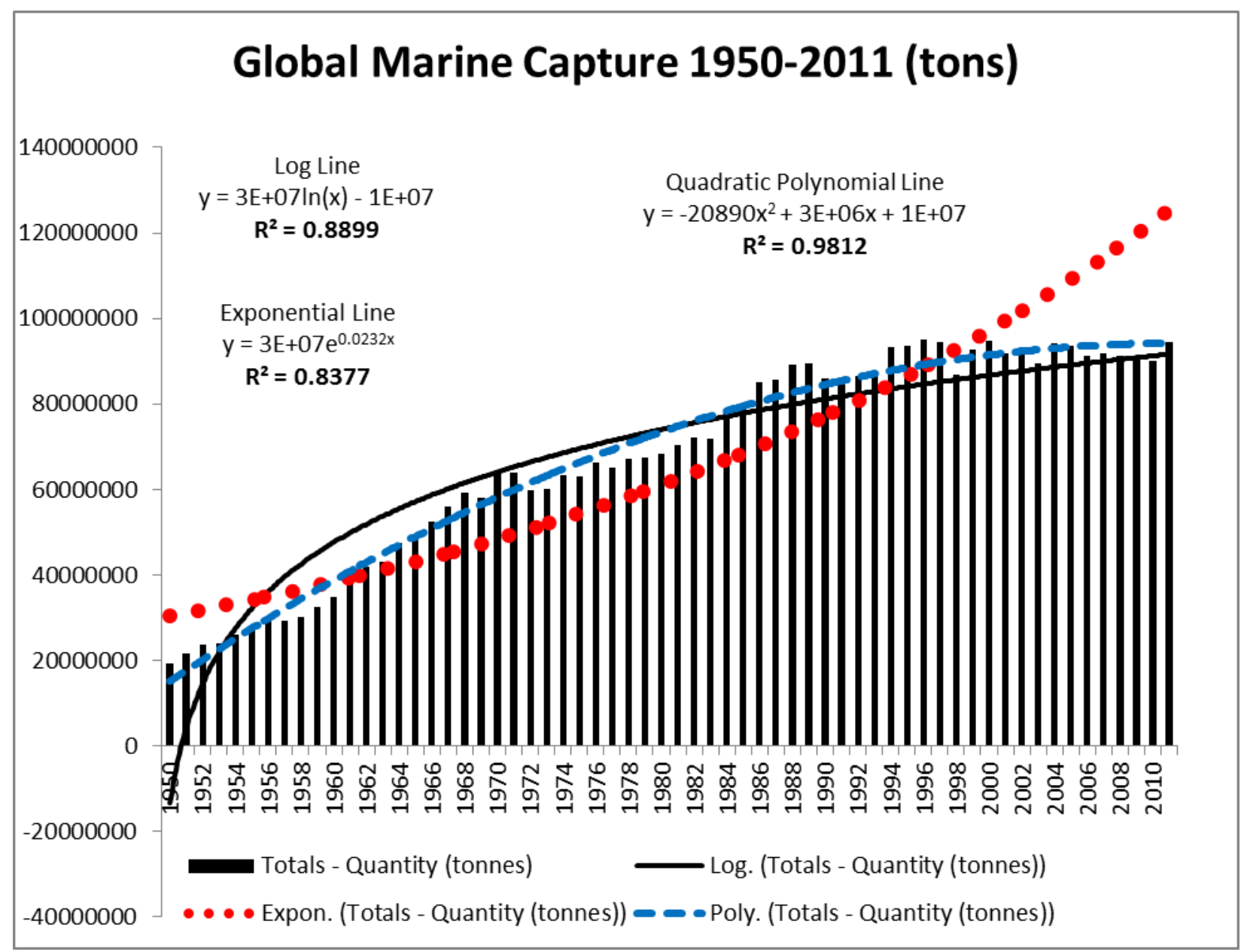

The point of trending the curve is not to be precise empirically about the fish catch, biomass, or some presumed global MSY, but rather to create a heuristic and a general proposition: the last of the fisheries that fished below MSY $(\sim 15 \%)$ are small, global catch effort has been steadily rising (below, and Part II), but global catch is roughly stable if not gradually receding and the graph in the long run (decades) will likely go down. Many stocks can be rebuilt to produce more particularly older industrialized fisheries in North America, Europe, and Oceana [5]. Meanwhile, "other parts of the world probably harbors higher but declining fish biomass on average, less control on exploitation rate, and less ability to set meaningful management capacity"[5]. Further, where fishing is forced to occur at deeper and deeper waters[6], but some of these fish, such as deepwater demersal fish, are inherently 
more susceptible to depletion given life histories that include a long maturity time and which allow for serial depletions[7].

Thus, the literature appears to support the notion of 1) fisheries as a series of nested adaptive cycles and 2) that the scale of this panarchy can plausibly be the collective world fisheries. If the world fisheries can be plausible described as a panarchy, then it is theoretically compelling that the world fisheries can and will follow the cycle phases. If world fisheries have entered the $\mathrm{K}$ phase, and if exploitation of the system continues unabated then it is plausible that the world fisheries system will cross a threshold or tipping point and enter rapidly into the release phase, $\Omega$. This tipping point would be a point at which there is no return to the old system, and the current world fishery system would enter into a "catastrophic shift" or state change [8]. The defining features of this state change are unpredictable, but it is implausible that it would be one of richer and more abundant fisheries, at least at first (until new speciation was able to be realized probably over thousands of years).

Unfortunately, the breakpoint in a world fisheries adaptive cycle cannot be precisely predicted base on the theory, though experiments in whole freshwater ecosystems indicate that there are statistical "early warning" signs[9]. Carpenter et al explain that, “... recent research has revealed statistical signals that precede some nonlinear transitions, such as rising autocorrelation, steep increases in variance, and extreme changes in skewness and shift in variance spectra toward low frequencies" (Ibid 1079). The variable that Carpenter et al monitored for these trends was chlorophyll, every five minutes for three years in two lakes - one manipulated and one control. These changes specifically can be initiated by structural perturbations, and it can be driven in fisheries by the addition or removal of top predators which, "destabilizes food webs, and extreme manipulations of predators cause trophic cascades, a type of regime shift that alters food web structure and ecosystem processes such as primary production, ecosystem respiration, and nutrient cycling" (Ibid). Unfortunately, monitoring the world ocean system (WOS) with this level of fine-grained data collection is impractical, though Boyce et al[10] have been able to synthesize ocean chlorophyll data going back to 1890 , and it may be possible to statistically analyze this chlorophyll data with changes in the marine food webs. Nonetheless, since the Carpenter, et al., experiment was done specifically with fish we can surmise that disruptions of the food web and substantial addition or removal of top ocean predators (TOPs) would be a precursor to nonlinear transitions.

Also, we know is that the length of the $\mathrm{K}$ phase will be determined by the "potential" and the "connectedness" in the cycle. For these elements, we turn to potential marine variables that may (or may not) determine this resilience.

\section{Other Ecosystem Factors}

From the above discussion, it is apparent that it is plausible if not likely that global fisheries in the WOS as a linked panarchy. This section will describe the possible variables in the WOS that may be important in thinking about the future of this panarchy, as well as the various scales. 
Changes in habitat abound in the ocean from changes to key ecosystem structures to changes in water column quality as a result of marine pollution. These are fundamental elements to the future of fisheries and are key variables for global fishery collapse.

Just as one example, coral reef ecosystems which provide habitat for "tens of thousands" of fish species and invertebrates have changed remarkably. While indigenous coastal cultures fished coral reefs for the last 35-40,000 years, this level of fishing appears to have had "limited ecological impact"(Jackson, 2001, p. 631). In particular, the acropora branching coral dominated the west Atlantic shallow reef ecosystems for millions of years, until as late as the 1980s when this coral "suffered sudden, catastrophic mortality in the 1980s due to overgrowth by macroalgae that exploded in abundance after mass mortality of the superabundant sea urchin Diadema antillarum that was the last remaining grazer of macroalgae" (Ibid). The Great Barrier Reef coral have faced numerous pressures, including an invasion of crown-of-thorns starfish that consumes coral, and this outbreak is likely to be a result of either overfishing species that consume the starfish larvae or nutrient overloading in the reef waters (Ibid). In the end, "Whales, dugongs, turtles, pearl oysters, and Trochus shell were each heavily exploited only to rapidly collapse, and all have failed to regain more than a small fraction of their former abundance" (Ibid). At this point, the prognosis for most coral reef ecosystems is grim. Coral face increase temperature pressure, where warming waters force bleaching and the migration of the animals that color and inhabit the hard coral structure (and in some cases come back afterward), disease, pollution, sediment runoff that suffocates the coral system, overfishing that removes grazers of algae, and physical destruction through tourism, fishing, coastal development, and ocean acidification all of which are geographically heterogenous even in places with high levels of spatial protection like the Great Barrier Reef which has lost $50 \%$ of its coral cover in under 30 years (Pandolfi et al., 2011, Silverman et al., 2009, De'ath et al., 2009, De'ath et al., 2012, Knowlton, 2012, DeGeorges et al., 2010, Fabricius, 2005, Hughes, 2003, Simpson, 2001, Stevens et al., 2000). At this point in history, the loss of coral reefs is a major factor in global biodiversity loss and is a good example of how biodiversity loss continues unabated, and the causes of biodiversity loss continue to strengthen (Butchart et al., 2010). Nearly one third of the world's coral face increased risk of extinction from these pressures (Carpenter et al., 2008).

Another key concern for marine habitats is pollution. These concerns cannot be thoroughly discussed here for space, but there has been a fundamental shift in marine pollution where we had been more concerned with the "usual suspects" of ocean dumping and this became the focus of international treaties, we now are facing marine challenges from "the invisibles"(Jacques, 2010a, Jacques, 2013). In particular, the invisibles are mostly natural elements: heat, $\mathrm{CO} 2$, nitrogen, and plastics. Plastics are the only artificial pollutant in this list, and one of the usual suspects that is of continued concern is human sewage, especially from growing megacities and slums which typically funnel sewage directly into the ocean. Briefly, since 1955 through 2010, the water column 0-2,000 meters warmed by $0.18^{\circ} \mathrm{C}$ because it absorbed $24 \times 1022 \mathrm{~J}$. Levitus writes, "If this heat were instantly transferred to the lower $10 \mathrm{~km}$ of the global atmosphere it would result in a...warming of this atmospheric layer by approximately $36^{\circ} \mathrm{C}$ $\left(65^{\circ} \mathrm{F}\right)$ "'(Levitus et al., 2012, p. L10603) and this warming is changing the mixing layers, increasingly stratifying the WOS and affecting the mechanisms for the Meridional Overturning Circulation (MOC)(Miles, 2009). Fishery production may increase in the higher latitudes from warming, but in 
lower latitudes the reduction of mixing may decline(Brander, 2007). Lehody(1997), in using El Nino as a proxy for future warming, found that two out of three studied tuna had reduced recruitment to adulthood, and in general warns that climate change in fisheries can and has created non-linear, abrupt changes to populations (Lehody et al., 2006). Thus warming will likely improve the productivity of some fisheries, and serious harm others, where in one study of 11 LMEs, some northern latitude pelagic fisheries were projected to increase $28-89 \%$ but other areas are expected to see "declines of $30-60 \%$ in potential fish production across some important areas of tropical shelf and upwelling seas, most notably in the eastern Indo-Pacific, the northern Humboldt and the North Canary Current"'(Blanchard et al., 2012, p. 2979).

Human emitted $\mathrm{CO} 2$ is a principal driver of this anthropogenic warming, but through the carbon cycle, most $\mathrm{CO} 2$ ends up in the ocean. Normally, the $\mathrm{CO} 2$ undergoes a chemical change to carbonate or bicarbonate, but as these compounds reach their saturation points, the $\mathrm{CO} 2$ remains carbonic acid, thus acidifying the water column faster than we have seen in 300 million years, reducing the $\mathrm{pH} 0.1$ since the Industrial Revolution(Fabry et al., 2008, Le Quesne and Pinnegar, 2012, Tyrrell, 2011, Bignami et al., 2013b). Research indicates that ocean acidification will likely threaten fish populations in subtle ways, altering "the potential to substantially influence the dispersal, survival, and recruitment of a pelagic fish species” (Bignami et al., 2013ap. 7366, Dixson et al., 2010, Munday et al., 2010).

Nitrogen is another natural element that is causing problems for biodiversity in the oceans. As a limiting element for growth, additions of $\mathrm{N}$ into delta regions from industrial agriculture, have rapidly increased episodic, seasonal, and permanent hypoxic, or "dead zones" so that there are now more than 400 of these hypoxic areas where marine life must leave or perish(Diaz and Rosenberg, 2008). Past research has shown that expanding hypoxic areas constrains tropical pelagic predatory fish into constrained bands of safe areas that increase their vulnerability to surface fishing gear(Prince and Goodyear, 2006). Hypoxia and changes in oxygen concentrations affects trophic interactions and mortality particularly of finfish and microinvertebrates that move, can challenge reproduction and growth, though analysis of 30 estuaries indicates local losses to fish catch probably do not translate to losses to the whole system except in places that lose critical habitat or where raw sewage if released (Breitburg et al., 2009). Thus, this is a serious pollution problem that reduces marine biodiversity(Robertson and Vitousek, 2009), but may not translate to a threat to global fisheries.

Finally, one of the new "invisibles" is marine plastic pollution, which photodegrades into micro pieces that float, but also absorb water and chemicals like PCBs, where they sink to a meter or so. This plastic is increasingly ubiquitous in both the coastal zones and gyres that accumulate plastic that has now entered the food chain, transporting toxic chemicals and taking up critical metabolic margins for marine animals (Barnes et al., 2009, Browne et al., 2011, Cole et al., 2011, Law et al., 2010, Ryan et al., 2009, Teuten et al., 2007). The system-wide effect of this pollution is unknown.

The above does not exhaustively cover all marine ecosystems or habitat changes, but it should be clear that critical life support systems for tens of thousands of species of fish and other marine life are and have been systematically degraded, with the most intensive changes occurring in contemporary period beginning in the latter 20th Century. While it is deeply uncertain to what degree or severity or scale 
these ecosystem changes have, we know that removing marine engineers that build the marine ecosystems, "could have devastating effects on local biodiversity and important water-sediment processes" that are compounded by biodiversity losses from fishing itself (Coleman and Williams, 2002, p. 40). Further, we know these changes challenge and stress fish populations rather than help rebuild them or make them more resilient. While obvious, this last point is non-trivial in this analysis because what is important for heuristic purposes are the directions of important variables across time that would make global fisheries more or less resilient.

\section{Conclusions}

What do we know and how do these factors affect the potential for global fishery collapse? We can surmise that global fisheries could potentially be a nested panarchy. This means it is likely for global populations to follow the phases, including collapse, of adaptive cycles. Collapse comes from the accumulation of slow changes that disturb the system. In addition to the fact that global fishery trends reflect a curve on its way down, we can see that there are many variables in play that would exacerbate the continued disturbance of over-fishing. From this research we can then infer that:

1. It is theoretically possible for global fisheries to collapse

2. The variables in fishery ecosystems are going in the wrong direction for resilience

3. Fish as food, and therefore food security, is in danger of catastrophic change putting millions of people at risk, to say nothing of the value of marine biodiversity in itself.

The ramifications of this conclusion are many. Fishery institutions at this point are built on national, bi-lateral, and multinational and regional settings, but are not managed globally. This poses a problem of fitness, or the way governing institutions fit the problem they are meant to solve and this may become a major problem to the effectiveness of international marine governance [11]. Global fisheries may also provide serious "leakage" problems where good regional management may limit fishing in one area but then those limited fishers may relocate to other regions. This is part of the "balloon" problem discussed by Barkin and DeSombre[12] who note that global management may be more effective. The metaphor is that when you push on a balloon, it pops out elsewhere; and, in fisheries, we may put managerial pressure on one area, only to find that the fishing pressure then changes to another area. Such a problem could be better coordinated at the scale that the problem exists - the global scale.

The threats to food security and marine biodiversity come together as fishing pressure creates serial depletions, particularly in the coastal areas, whereby fishers have to go to deeper and deeper water while fish must survive in a dramatically changing water column. It may be possible to form rules that have the twin goals of increasing food security and protecting marine biodiversity through management more closely aligned with holistic ecosystem thinking. However, even though we know that approach is both biologically and socially more sound, few regional fishery management organizations (RFMOs) are using it[13].

Clearly, the world's oceans are in crisis, and international management of fisheries has largely failed. Even if global fishery collapse is highly unlikely, we cannot be so naïve as to think that the combination of ecological crisis and governing failure are a recipe for a sound future where people can rely on a steady and affordable diet of fish. Since the coastal poor rely on fish for food security and are the most vulnerable to changes, they will be and are the worst off (perhaps except for the fish themselves) because of these trends. This vulnerability will probably intensify further as coastal fisheries that are more available to the coastal poor are depleted, and fish becomes more of a global 
commodity traded to the highest bidder. These are all very serious reasons for thinking more holistically about the way we manage fish at the global level.

\section{Conflict of Interest}

The author declares no conflict of interest.

\section{References and Notes}

1. Holling, C.S., Resilience and stability of ecological systems. Annu. Rev. Ecol. Syst. 1973, 265, 1547-1551.

2. Holt, S., Sunken billions - but how many? Fisheries Research 2009, 97, 3-10.

3. Willman, R.; Kelleher, K.; Arnason, R.; Franz, N., The sunken billions: The economic justification for fisheries reform. World Bank/United Nations Food and Agriculture Organization: Washington, D.C., 2008.

4. FAO State of the world fisheries and aquaculture; Food and Agriculture Organization of the United Nations: Rome, 2012.

5. Worm, B.; Branch, T.A., The future of fish. Trends in Ecology \&amp; Evolution 2012.

6. Morato, T.; Watson, R.; Pitcher, T.J.; Pauly, D., Fishing down the deep. Fish Fish. 2006, 7, $24-$ 34.

7. Cheung, W.W.; Watson, R.; Morato, T.; Pitcher, T.J.; Pauly, D., Intrinsic vulnerability in the global fish catch. MARINE ECOLOGY-PROGRESS SERIES- 2007, 333, 1.

8. Scheffer, M.; Carpenter, S.; Foley, J.; Folke, C.; Walker, B., Castrophic shifts in ecosystems. Nature 2001, 413, 591-596.

9. Carpenter, S.; Cole, J.; Pace, M.; Batt, R.; Brock, W.; Cline, T.; Coloso, J.; Hodgson, J.; Kitchell, J.; Seekell, D., Early warnings of regime shifts: A whole-ecosystem experiment. Sci 2011, 332, 1079-1082.

10. Boyce, D.G.; Lewis, M.; Worm, B., Integrating global chlorophyll data from 1890 to 2010. Limnol. Oceanogr. Methods 2012, 10, 840-852.

11. Young, O.R., Effectiveness of international environmental regimes: Existing knowledge, cutting-edge themes, and research strategies. Proceedings of the National Academy of Sciences 2011, 108, 19853-19860.

12. Barkin, J.S.; DeSombre, E.R., Saving global fisheries: Reducing fishing capacity to promote sustainability. Mit Press: 2013.

13. Webster, D.G., International fisheries: Assessing the potential for ecosystem management. Journal of Environmental Studies and Sciences 2013, 3, 169-183.

(C) 2013 by the author; licensee MDPI, Basel, Switzerland. This article is an open access article distributed under the terms and conditions of the Creative Commons Attribution license (http://creativecommons.org/licenses/by/3.0/). 